\title{
Patrones de distribución de alturas de bosques antiguos siempreverde del centro-sur de Chile
}

\author{
Tree height distributions in the canopy of old-growth temperate rainforests \\ of south-central Chile
}

\author{
Alejandro Oyarzún a, Pablo J Donoso a, Álvaro G Gutiérrez b* \\ ${ }^{\text {a } U n i v e r s i d a d ~ A u s t r a l ~ d e ~ C h i l e, ~ F a c u l t a d ~ d e ~ C i e n c i a s ~ F o r e s t a l e s ~ y ~ R e c u r s o s ~ N a t u r a l e s, ~}$ \\ Instituto de Bosques y Sociedad, Valdivia, Chile, pdonoso@uach.cl. \\ *Autor de correspondencia: ${ }^{b}$ Universidad de Chile, Facultad de Ciencias Agronómicas, \\ Departamento de Ciencias Ambientales y Recursos Naturales Renovables, Santiago, Chile, bosqueciencia@gmail.com
}

\begin{abstract}
SUMMARY
Canopy structure is relevant to understand forests functioning and sustaining biodiversity. In Chile, old-growth temperate evergreen rainforests have been characterized by a muti-stratified canopy; however, it is uncertain the common number of strata formed in the canopy or how dynamic processes structure the forest canopy. In this study, we evaluated two alternative hypotheses to explain canopy structure in old-growth temperate evergreen rainforests of Chile, i) the increased density of shade-tolerant tree species along the succession should lead to a non-stratified canopy, and ii) the occurrence of disturbances (e.g. individual tree falls opening gaps in the canopy) would allow species with different life strategies to concentrate at specific canopy heights, leading to stratified canopies. We tested these hypotheses in four old-growth stands, where we measured tree height distributions and the degree of correlation among vertical variables. We found two to three well defined strata in the canopy made up of species groups with similar functional traits. These results support the second hypothesis and suggest that the pattern of crown aggregation may be explained by the life strategies of the species, adapted to the occurrence of canopy openings. The dynamics of canopy gaps favors a stable canopy stratification in these forests along the succession. This is relevant for the elaboration of sustainable management strategies and the conservation of the biodiversity of these ecosystems.
\end{abstract}

Key words: vertical distribution, stratification, heterogeneity, life strategies, dynamic processes.

\section{RESUMEN}

La estructura vertical del dosel es determinante en el funcionamiento de los bosques y el sostenimiento de la biodiversidad. En Chile, los bosques antiguos del tipo forestal siempreverde han sido caracterizados con una estructura de dosel mutiestratificado, no obstante, no existe certeza del número de estratos que se forman, ni cómo los procesos dinámicos influyen en esta estructuración. En este estudio se exploraron dos hipótesis alternativas para explicar la estructuración del dosel en bosques antiguos siempreverdes, i) la densidad de especies arbóreas tolerantes a la sombra propician un dosel no estratificado, y ii) la ocurrencia de disturbios (e.g. caídas individuales de árboles), permitiría que especies con distintas estrategias de vida se concentren en alturas específicas del dosel, propiciando doseles estratificados. Se pusieron a prueba estas hipótesis en cuatro bosques antiguos del tipo forestal siempreverde, donde se midieron las distribuciones de alturas de árboles y el grado de correlación entre variables verticales. Se encontraron dos a tres estratos en el dosel, cada uno conformado por grupos de especies con similares rasgos funcionales. Estos resultados apoyan la segunda hipótesis y sugieren que el patrón de agregación de copas en estratos definidos, sería explicado por las estrategias de vida de las especies, adaptadas a la ocurrencia de claros en el dosel. La dinámica de claros favorece una estratificación estable del dosel de estos bosques a lo largo de su desarrollo, aspecto relevante a considerar para la elaboración de estrategias de manejo sostenible y la conservación de la biodiversidad de estos ecosistemas.

Palabras clave: distribución vertical, estratificación, heterogeneidad, estrategias de vida, procesos dinámicos.

\section{INTRODUCCIÓN}

El dosel del bosque tiene un rol fundamental sobre las funciones ecológicas de los ecosistemas forestales. La fotosíntesis de las plantas ocurre en el dosel del bosque, lo que determina la productividad y captura de carbono en estos ecosistemas (Hardiman et al. 2013). El dosel también sostiene la biodiversidad a través de variados procesos ecológicos (e.g. interacciones planta-animal, polinización), y provee refugio para la flora y fauna de estos 
ecosistemas (Díaz et al. 2005). Por lo tanto, comprender los factores que determinan la estructura del dosel es fundamental para la toma de decisiones respecto a la conservación y manejo de los bosques (Franklin et al. 2002).

La estructura del dosel está definida por la distribución vertical y espacial de los árboles, las dimensiones de sus copas y la arquitectura de las plantas que lo componen (e.g. árboles, arbustos, plantas epífitas, lianas). En el caso de la estructura vertical del dosel (e.g. distribución de las alturas de los árboles; Donoso 1993), existe un patrón funcional o fisiológico que determina el posicionamiento en altura de los árboles, donde las especies de mayor demanda lumínica ocupan la parte alta del dosel, y las especies de menor demanda de luz se posicionan en la parte baja del dosel (Terborgh 1985). Sin embargo, a lo largo de su desarrollo, los bosques presentan cambios estructurales (e.g. aberturas de dosel producidas por la muerte o caída árboles), que inciden en la intensidad lumínica a través del perfil del bosque (Oliver y Larson 1996, Franklin et al. 2002). El tipo y magnitud de estos cambios está determinado por la variabilidad espacial y temporal en la ocurrencia de claros que, entre otros factores, afecta la regeneración de especies arbóreas de distinta tolerancia a la sombra (Franklin y Van Pelt 2004, Gutiérrez et al. 2004). En consecuencia, el proceso de desarrollo del bosque y los procesos dinámicos que ocurren en él, producen el recambio de cohortes de árboles con distintos rasgos funcionales (e.g. tasas de crecimiento, alturas máximas, tolerancia a la sombra), que generan cambios en dos características importantes de la estructura vertical como lo son la estratificación y la heterogeneidad del dosel del bosque.

Se puede definir la estratificación vertical del dosel como la concentración de las copas de los árboles en una o varias alturas específicas dentro del dosel, que obedece a la formación de campos lumínicos al interior del bosque, donde las diferentes especies se posicionan para satisfacer sus necesidades biológicas (Terborgh 1985). Según Terborgh (1985) diversos factores influirían en la conformación de los estratos del dosel, tales como la composición de especies, densidad de individuos y la latitud donde se encuentra los bosques. Por otro lado, la heterogeneidad vertical, corresponde al grado de distribución o dispersión de los árboles a diferentes alturas dentro del dosel, la cual sufre cambios a medida que ocurre mortalidad de árboles y nuevos individuos acceden a doseles superiores del bosque (Franklin y Van Pelt 2004). De esta manera, doseles con presencia de individuos ubicados a variadas alturas dentro del perfil vertical (i.e. doseles no estratificados), son más heterogéneos que doseles con una gran cantidad de individuos ubicados a una altura similar dentro del perfil del bosque.

En Chile se han realizado escasos estudios enfocados en la estructura vertical del dosel, que sumado al tipo de estudio y amplio rango geográfico que existe entre estos, no permiten tener una certeza de los patrones de distribución vertical del dosel (e.g. número de estratos arbó- reos), ni de cómo los procesos dinámicos participan en la configuración de este (e.g. influencia de disturbios). Por ejemplo, Donoso (1993) señala que los bosques del tipo forestal siempreverde de Chile poseen un dosel multiestratificado, sin embargo, esta definición no aclara cuantos estratos tienen efectivamente estos bosques. Lusk y Ortega (2003), exponen que en bosques secundarios de la zona centro sur de Chile, luego del restablecimiento de especies pioneras, se forman doseles monotípicos y unimodales, al cual van accediendo nuevas cohortes de especies tolerantes a la sombra que forman un segundo estrato arbóreo, que aportan al área basal del bosque. Además, estos autores sugieren que a medida que algunos árboles superan el dosel estos contribuyen a que los bosques tengan más alta biomasa, por ejemplo, debido al uso complementario de la luz. Por otra parte, contrariamente al concepto de dosel multiestratificado, Gutiérrez et al. (2004) reportan una distribución de dosel unimodal no estratificada en bosques antiguos de Chiloé, posiblemente propiciado por la ausencia de disturbios catastróficos, dando cuenta que los bosques en sucesión tardía pueden presentar este tipo de distribución. Estos resultados documentan la variabilidad de la estructura vertical de bosques siempreverdes de Chile y la necesidad de entender los patrones de distribución de altura de las copas de los árboles para diversos rodales de este tipo forestal. Esto es particularmente relevante de discernir en bosques adultos con escasa perturbación humana, ya que son ecosistemas diversos en especies, que aportan diversos servicios ecosistémicos, almacenan grandes cantidades de carbono y continúan capturando dióxido de carbono de la atmósfera (Watson et al. 2018).

Dado que el proceso de desarrollo del bosque aumenta la heterogeneidad vertical y una ocupación completa del dosel arbóreo por parte de especies tolerantes a la sombra, es posible que existan doseles no estratificados en bosques antiguos siempreverde. No obstante, bosques de sucesión tardía muy comúnmente presentan doseles con múltiples estratos de altura (Terborgh 1985, Ashton y Hall 1992, Oliver y Larson 1996). Ya que en los bosques existe la posibilidad de encontrar tanto doseles sin estratificación como doseles estratificados (Franklin y Van Pelt 2004), se exploraron dos hipótesis posibles que pueden explicar la presencia o no, de estratos diferenciados en el perfil vertical de los bosques antiguos siempreverde de Chile: i) la heterogeneidad de dosel generada por la dominancia de especies arbóreas tolerantes a la sombra propicia un dosel no estratificado; y ii) la ocurrencia de disturbios de diferente escala y magnitud, permite que árboles de especies con diferentes estrategias de vida se concentren en diferentes alturas del dosel propiciando estructuras con estratos definidos en el perfil vertical del bosque. Para explorar estas hipótesis, este estudio busca i) verificar si las distribuciones de alturas en bosques antiguos se encuentran o no estratificadas; y ii) determinar cómo los procesos dinámicos y las características verticales del bosque influyen en la estructuración del dosel. 


\section{MÉTODOS}

Bosques estudiados. Se analizaron cuatro bosques antiguos del tipo forestal siempreverde ubicados en la zona centro sur de Chile (cuadro 1). Se buscaron signos en los bosques in situ para comprobar si los bosques no tenían evidencias de cortas (e.g. presencia de tocones) o incendios recientes (e.g. presencia de cicatrices de fuego), y que tuvieran atributos estructurales de un estado sucesional avanzado, tales como presencia de grandes árboles, dosel parchoso incluyendo claros, estructura en la que todas las clases de tamaño estaban representadas, entre otros (Gutiérrez et al. 2009).

Medición de la estructura vertical. En cada uno de los rodales se instalaron parcelas (cuadro 1), donde se midió el diámetro a $1,5 \mathrm{~m}$ de altura de fuste (dap), la altura total y de todos los árboles (incluido el registro de la especie) con dap $\geq 5 \mathrm{~cm}$, utilizando cinta diamétrica e hipsómetro de ultrasonido VERTEX Haflog ${ }^{\circledR}$, respectivamente.

Análisis estadístico. Se ajustó un modelo de mixtura gaussiana a las distribuciones de alturas de cada rodal. Este método permite representar situaciones no paramétricas debido a su flexibilidad de modelamiento (McLachlan y Peel 2000, ecuación 1):

$$
f(x)=\sum_{i=1}^{k} w_{i} \emptyset\left(x \mid u_{i}, \sigma_{i}\right)
$$

Donde: $f(x)$ es una suma ponderada de $k$ densidades normales, donde la proporción de mixtura o peso es $w_{i} \mathrm{y}$ $\varnothing\left(x_{i} \mid u_{i}, \sigma_{i}\right)$ es una densidad normal de media $u_{i}$ y varianza $\sigma_{i}^{2}$ del i-esimo componente con peso $w_{i}$.

Cuadro 1. Características de los bosques antiguos estudiados.

Characteristics of the studied old-growth forests.
Se estimaron los parámetros del modelo mediante el procedimiento de cadenas reversibles de Markov Montecarlo $(\mathrm{MCMC})$ y se determinó el número de modas por medio de la función propuesta por Xu et al. (2014). Para el ajuste se utilizó el paquete "mixAK" (Komárek 2009) del programa estadístico R (R Development Core Team 2017). Este modelo tiene el potencial de cuantificar objetivamente el número de modas del conjunto de datos analizado (Xu et al. 2014).

Características estructurales verticales. Posterior a la evaluación de la estratificación de los rodales, se determinaron los parámetros estructurales de altura máxima del dosel, altura promedio del dosel, altura de las posibles estratificaciones y heterogeneidad vertical del dosel. Para este último cálculo, en primer lugar se crearon grupos o clases de alturas (e.g. 0-6, 6-12, 12-18, etc.) los cuales se definieron por la siguiente división, $n /(\operatorname{Hmax} / \sigma)$, donde $n$ es número total de datos de la parcela, Hmax es la altura máxima del dosel de la parcela y $\sigma$ es la desviación estándar de la distribución de alturas de la parcela. Posteriormente, se utilizó el inverso del índice de diversidad (d) de Berger-Parker (Gutiérrez et al. 2009, ecuación 2). Este índice ha sido utilizado para analizar el nivel de heterogeneidad vertical en bosques siempreverdes, donde valores cercanos a 1 indican bosques con dosel homogéneo, por el contrario, valores cercanos a 3 indica doseles heterogéneos (Gutiérrez et al. 2009).

$$
d=\frac{n}{N \max }
$$

Donde, $n$ es el número total de individuos, y Nmax es el número de individuos del grupo de datos más abundante (clase de altura con más individuos).

\begin{tabular}{lcccc}
\hline \multirow{2}{*}{ Características de sitio } & \multicolumn{3}{c}{ Rodal } \\
\cline { 2 - 5 } & Correntoso & Llancahue A & Llancahue B & Puyehue \\
\hline Coordenadas geográficas & $41^{\circ} 30^{\prime} 59^{\prime \prime} \mathrm{S}$ & $39^{\circ} 50^{\prime} 21^{\prime \prime} \mathrm{S}$ & $39^{\circ} 50^{\prime} 19^{\prime \prime} \mathrm{S}$ & $40^{\circ} 38^{\prime} 60^{\prime} \mathrm{S}$ \\
Altitud (m s.n.m.) & $72^{\circ} 39^{\prime} 10,02^{\prime} \mathrm{W}$ & $73^{\circ} 07^{\prime} 46^{\prime} \mathrm{W}$ & $73^{\circ} 08^{\prime} 03^{\prime} \mathrm{W}$ & $72^{\circ} 10^{\prime} 60^{\prime \prime} \mathrm{W}$ \\
Exposición & $384-470$ & 360 & 326 & 448 \\
Pendiente (\%) & $\mathrm{NE}$ & $\mathrm{NW}$ & $\mathrm{NW}$ & $\mathrm{SW}$ \\
Superficie medida $\left(\mathrm{m}^{2}\right)$ & $10-30$ & $10-30$ & $10-40$ & $10-30$ \\
$\mathrm{~N}^{\circ}$ de parcelas & 3500 & 2025 & 3500 & 5000 \\
\left.${\text { Área basal }\left(\mathrm{m}^{2} \text { ha }\right.}^{-1}\right)$ & 7 & 3 & 7 & 8 \\
$\mathrm{~N}^{\circ}$ árboles medidos & 102,3 & 79,9 & 108,4 & 140,8 \\
Altura dosel dominante $(\mathrm{m})$ & 390 & 258 & 404 & 289 \\
\hline
\end{tabular}


El cálculo de los parámetros se realizó con la información de las 25 parcelas medidas, de modo que el valor de la variable en cada bosque se obtuvo a partir del promedio de los valores obtenidos de las parcelas muestreadas en cada uno de ellos. Además, se determinó el grado de estratificación de cada una de estas, y posteriormente, se analizó el grado de asociatividad entre un grupo de 20 variables estructurales por el método de correlación de Pearson, donde valores cercanos a 1 reflejan una relación positiva fuerte y valores cercanos a -1 una relación negativa fuerte, y se seleccionaron aquellas correlaciones que explicaran de mejor manera las variaciones estructurales verticales de los rodales.

Análisis de distribuciones de alturas de árboles por especie. Se realizó un análisis de agrupamiento o cluster jerárquico basado en las distribuciones de alturas de los árboles por especies, con el fin identificar los posibles grupos que se puedan formar en el dosel y que expliquen la distribución general de alturas del rodal. Para realizar este análisis se construyó una matriz, donde por medio del test de Kruskal-Wallis se compararon las distribuciones de alturas de todas las especies con tamaño muestral mayor a 10 (cuadro 2). Las similitudes entre los valores de Kruskal-Wallis fueron calculadas mediante la correlación de Spearman y el clúster de datos con el algoritmo de distancia máxima. Luego, se testearon las agrupaciones que formaron las especies por medio del método K-means, con el número óptimo de clúster determinado por el método de Silhouette. Estos grupos se graficaron por medio de la función de densidad de kernel, calculando sus respectivos coeficientes de asimetría (Sk).

En este análisis se utilizó la base de datos completa, dado que todos los bosques tienen edades sucesionales similares (sensu Gutiérrez y Huth 2012) y comparten el mismo tipo forestal. Adicionalmente, en la fase de exploración de datos se verificó que las especies exhibieran rangos de distribuciones de altura de árboles que no presentaran diferencias evidentes entre los distintos bosques estudiados.

Cuadro 2. Tolerancia a la sombra de especies arbóreas según Gutiérrez et al. (2004, 2009). Especies con una muestra $>10$ individuos. Alturas máximas según datos recopilados para este estudio, y Gutiérrez y Huth (2012). Longevidad máxima según 1: Pollmann y Veblen (2004) 2: Gutiérrez y Huth (2012) y datos recopilados para este estudio. El cuadro esta ordenado según altura máxima de la especie.

Shade tolerance of tree species with a sample $>10$ trees according to Gutiérrez et al. (2004, 2009). Maximum heights according to data collected for this study, and Gutiérrez and Huth (2012). Maximum longevity according to 1: Pollmann and Veblen (2004) 2: Gutiérrez and Huth (2012) and data collected for this study. The table is sorted according to the maximum height of the species.

\begin{tabular}{|c|c|c|c|c|c|}
\hline Especie & $\begin{array}{l}\text { Tamaño } \\
\text { muestral }\end{array}$ & Código & Tolerancia a la sombra & $\begin{array}{c}\text { Altura } \\
\text { máxima }(\mathrm{m})\end{array}$ & $\begin{array}{l}\text { Longevidad } \\
\text { máxima (años) }\end{array}$ \\
\hline Crinodendron hookerianum Gay & 12 & Crho & Intermedio & 11 & Sin datos \\
\hline Myrceugenia exsucca (DC.) O. Berg & 10 & Myex & Tolerante & 11 & Sin datos \\
\hline Rhaphithamnus spinosus (Juss.) Moldenke & 14 & Rhsp & Tolerante-Intermedio & 15 & Sin datos \\
\hline Lomatia dentata (Ruiz et Pav.) R. Br. & 19 & Lode & Tolerante & 17 & Sin datos \\
\hline Lomatia ferruginea (Cav.) R. Br. & 12 & Lofe & Intermedio & 22 & Sin datos \\
\hline Gevuina avellana Molina & 30 & Geav & Intermedio & 24 & Sin datos \\
\hline Amomyrtus luma (Molina) D. Legrand et Kausel & 109 & Amlu & Tolerante & 26 & $200^{2}$ \\
\hline Caldcluvia paniculata (Cav.) D. Don & 52 & Capa & Tolerante-Intermedio & 26 & $117^{2}$ \\
\hline Amomyrtus meli (Phil.) D. Legrand et Kausel & 27 & Amme & Tolerante-Intermedio & 26 & Sin datos \\
\hline Myrceugenia planipes (Hook. et Arn.) O. Berg & 60 & Mypl & Tolerante & 29 & $260^{2}$ \\
\hline Drimys winteri J.R. Forst. et G. Forst. & 102 & Drwi & Intermedio-Intolerante & 34 & $250^{2}$ \\
\hline Dasyphyllum diacanthoides (Less.) Cabrera & 35 & Dadi & Intermedio-Intolerante & 38 & $331^{2}$ \\
\hline Saxegothaea conspicua Lindl. & 31 & Saco & Tolerante & 38 & $750^{2}$ \\
\hline Aextoxicon punctatum Ruiz et Pav. & 177 & Aepu & Tolerante & 45 & $480^{2}$ \\
\hline Laureliopsis philippiana (Looser) Schodde & 506 & Laph & Tolerante & 45 & $657^{2}$ \\
\hline Nothofagus nitida (Phil.) Krasser & 19 & Noni & Intolerante & 52 & $600^{2}$ \\
\hline Eucryphia cordifolia Cav. & 74 & Euco & Intermedio-Intolerante & 51 & $650^{2}$ \\
\hline Nothofagus dombeyi (Mirb.) Oerst. & 19 & Nodo & Muy Intolerante & 53 & $780^{1}$ \\
\hline
\end{tabular}




\section{RESULTADOS}

Estructura vertical. Los resultados del ajuste del modelo de mixturas y test de multimodalidad arrojaron dos modas en las distribuciones de alturas para Correntoso, Llancahue A y B, mientras que Puyehue presentó tres modas (cuadro 3). Se observó que el modelo de mixtura gaussiana replicó razonablemente bien la distribución de alturas expresada por los histogramas (figura 1), potencialmente indicando que todos los bosques se encuentran estratificados.
La altura del estrato o moda inferior de los bosques en general se expresó entre los 9 y 12 m (cuadro 4), siendo más densa y menos variable que la moda superior, que se ubicó entre los 17 y los $35 \mathrm{~m}$ de altura (figura 1, cuadro 4). Solo en Puyehue se encontró un estrato intermedio a los $25 \mathrm{~m}$ de altura aproximadamente, siendo también el rodal de mayor altura promedio. En cuanto a la heterogeneidad, todos los bosques presentaron valores sobre 2,1 , siendo Correntoso con un valor del índice de Berger-Parker de 2,5 , el rodal con una mayor heterogeneidad vertical. El

Cuadro 3. Valores de probabilidad de número de modas en cada bosque. *Número de modas determinado por el máximo valor de probabilidad de la prueba de multimodalidad.

Probability values of number of modes in each forest. *Number of modes determined by the maximum probability value of the multimodality test.

\begin{tabular}{lcccccc}
\hline \multirow{2}{*}{ Rodal } & $\mathrm{N}$ & \multicolumn{5}{c}{ Modas } \\
\cline { 3 - 7 } & & 1 & 2 & 3 & 4 & 5 \\
\hline Correntoso & 390 & 0,34 & $0,48^{*}$ & 0,15 & 0,03 & 0,01 \\
Llancahue A & 258 & 0,04 & $0,51^{*}$ & 0,30 & 0,13 & 0,04 \\
Llancahue B & 404 & 0,04 & $0,49^{*}$ & 0,31 & 0,12 & 0,04 \\
Puyehue & 289 & 0,01 & 0,14 & $0,47^{*}$ & 0,27 & 0,09 \\
\hline
\end{tabular}
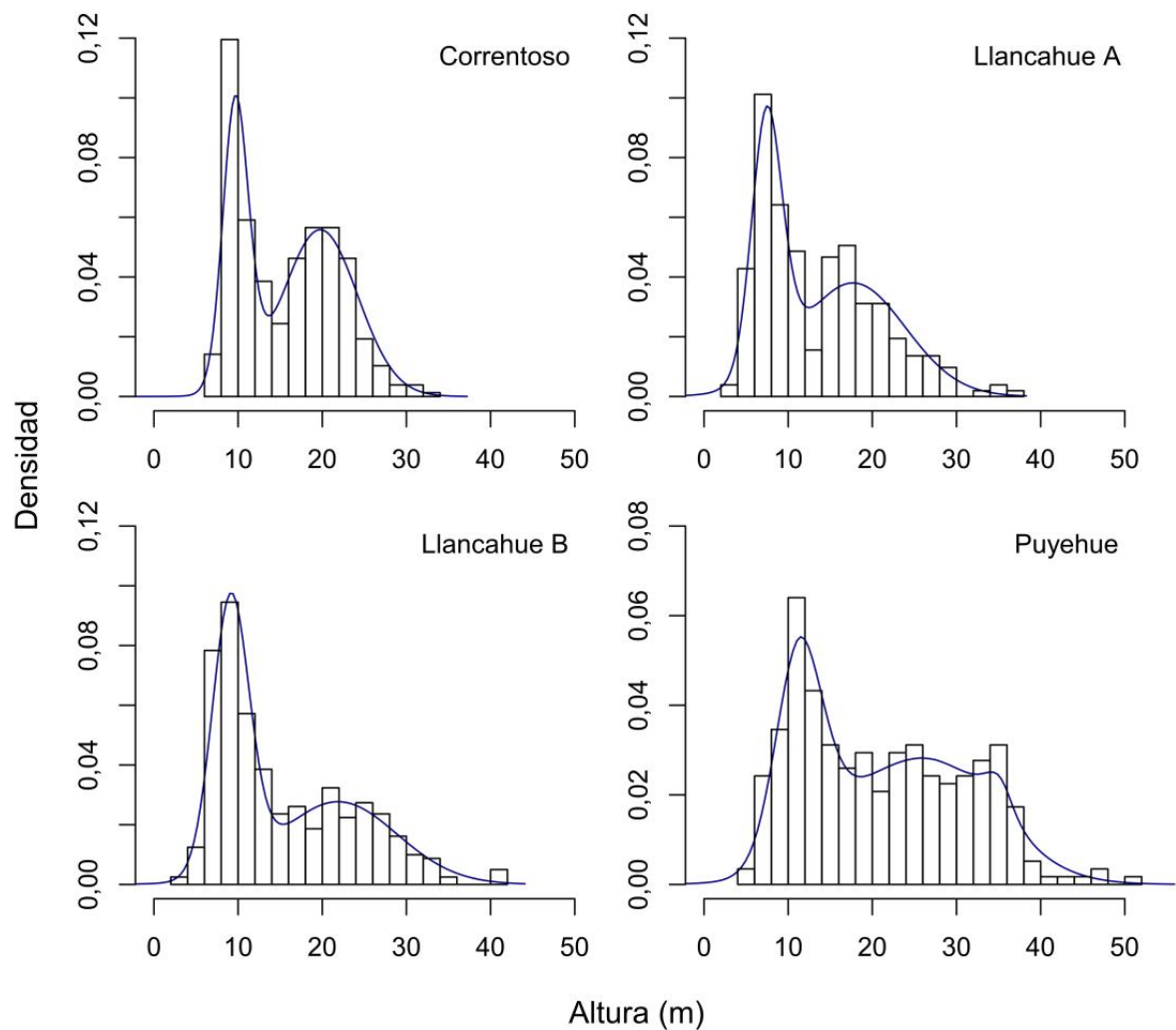

Figura 1. Distribuciones de alturas de los bosques estudiados. La línea indica la distribución del modelo de mixtura ajustado a los datos. Height distributions of the studied forests. The line indicates the distribution of the mixed model adjusted to the data. 
porcentaje de árboles tolerantes a la sombra se encontró en todos los bosques alrededor del $80 \%$, siendo Llancahue B el rodal con mayor presencia de árboles tolerantes $(87 \%$; cuadro 4). Por otra parte, el número de estratos se expresó de manera diferente a nivel de parcela que a nivel de rodal. A escala de las parcelas se observó que Puyehue no presentó tres estratos, mientras que en los demás bosques si se observaron parcelas con tres estratos arbóreos (cuadro 4).

Los resultados de la correlación de Pearson, arrojaron una relación negativa entre la heterogeneidad y el número de estratos (cuadro 5), indicando que existe un aumento en la heterogeneidad del dosel en función de la disminución del número de estratos (parcelas con tres estratos presentaron una media en el índice de Berger-Parker de 1,7). De la misma manera, se encontró una relación positiva entre el número de modas y el número de árboles emergentes, lo que significó que se observaran hasta tres estratos en función del aumento de árboles emergentes, aunque también dependió de que estos árboles fueran de gran altura y que sobrepasaran por más de $5 \mathrm{~m}$ al dosel dominante (figura 1). Por otro lado, se obtuvo una correlación negativa entre el número de árboles emergentes y el porcentaje de árboles tolerantes a la sombra, indicando que existe una disminución de árboles emergentes a medida que aumenta el porcentaje de árboles tolerantes a la sombra. Finalmente, las alturas de las modas 1 y 2 , variaron en función de la altura del rodal, ya que la moda 1 (inferior) significó un aumento de la altura en función de la altura de la moda 2 (superior), y a la variación de la tolerancia a la sombra de las especies que dominan el rodal.

Agrupación de especies. El análisis de clúster indicó que las especies formaron cuatro agrupaciones de acuerdo a la similitud de sus distribuciones de alturas (figura 2), aunque se observó una importante y primera diferenciación entre el grupo de las especies más intolerantes a la sombra (grupos c y d) y el grupo de especies con mayor tolerancia a la sombra (grupos a y b).

Complementariamente, los tipos de distribuciones de densidad de altura de los clústers de especies, dieron cuenta de las claras diferencias que existen entre ellos, ya que presentaron tipos de distribuciones de alturas que obede-

Cuadro 4. Valores de las variables estructurales del dosel de los rodales estudiados.

Values of the structural variables of the canopy in the studied stands.

\begin{tabular}{lcccc}
\hline Variables estructurales & Correntoso & Llancahue -A & Llancahue -B & Puyehue \\
\hline Berger-Parker (d) & 2,4 & 2,2 & 2,1 & 2,3 \\
Altura máxima del dosel (m) & 29,7 & 31,9 & 35,9 & 42,3 \\
Altura promedio del dosel (m) & 16,2 & 13,8 & 15,4 & 21,3 \\
Moda inferior rodal (m) & 9,5 & 7,6 & 9,1 & 12,1 \\
Moda media rodal (m) & - & - & - & 25 \\
Moda superior rodal (m) & 20,5 & 16,3 & 23,5 & 35 \\
Tolerantes (\%) & 74 & 78 & 87 & 82 \\
Parcelas con 1 estrato & 2 & 0 & 0 & 4 \\
Parcelas con 2 estratos & 5 & 2 & 5 & 4 \\
Parcelas con 3 estratos & 1 & 1 & 2 & 0 \\
\hline
\end{tabular}

Cuadro 5. Valores de coeficiente de Pearson de variables verticales correlacionadas.

Pearson coefficient values of correlated vertical variables.

\begin{tabular}{lcl}
\hline Variables correlacionadas & Correlación de Pearson & $P$ \\
\hline Berger-Parker $\sim \mathrm{N}^{\circ}$ modas & $-0,62$ & $0,002 * *$ \\
$\mathrm{~N}^{\circ}$ modas $\sim \mathrm{N}^{\circ}$ emergentes & 0,62 & $0,020^{*}$ \\
$\mathrm{~N}^{\circ}$ emergentes $\sim \%$ tolerantes dominantes & $-0,51$ & $0,004 * *$ \\
Altura moda $1 \sim \%$ tolerantes dominantes & 0,61 & $0,004 * *$ \\
Altura moda $1 \sim$ Altura moda 2 & 0,57 & $0,008 * *$ \\
Altura moda $2 \sim$ Altura máxima & 0,65 & $0,002 * *$ \\
\hline
\end{tabular}


cen a los diferentes rasgos de tamaño, longevidad y tolerancia a la sombra de cada grupo y que consecuentemente determinan su posición en el dosel (tamaño + longevidad + tolerancia: $\mathrm{R}^{2}=0,7, P=0,0016$ ) (figura 3 ). En este sentido, las especies pertenecientes al dosel inferior (grupo "a"), funcionalmente tolerantes y medianamente tolerantes a la sombra, adoptaron distribuciones de asimetría positiva (Sk cercanos a 1, figura 3). Las especies que se distribuyen en todo el dosel (grupo "b"), todas tolerantes a la sombra, presentaron una distribución bimodal en Correntoso, una distribución asimétrica negativa en Llancahue y una distribución normal en Puyehue (Sk cercano a 0). El grupo que se distribuye principalmente en el dosel superior (grupo "c"), se encontró compuesto mayoritariamente por

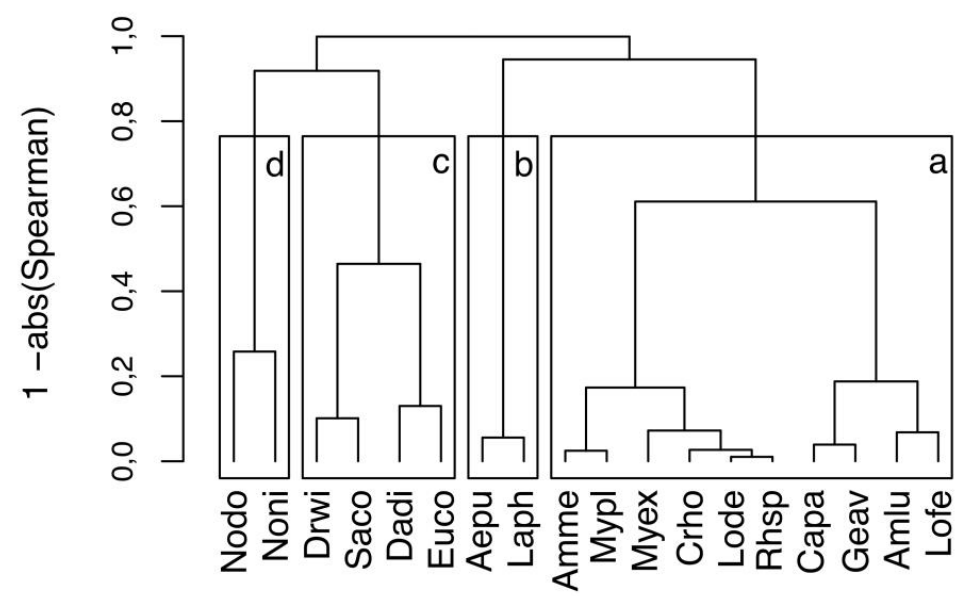

Figura 2. Dendrograma de las distribuciones de alturas de árboles de las especies de los bosques estudiados. Cuadros negros señalan número de grupos óptimos indicados por el método de Silhouette. El eje y corresponde a los valores absolutos de correlación Spearman (abs (Spearman)). Codificación de especies acorde al cuadro 2.

Dendrogram of the tree height distributions in the studied forests. Black tables show the number of optimal groups indicated by Silhouette's method. The y-axis corresponds to the absolute values of Spearman correlation (abs (Spearman)). Species coding according to table 2.

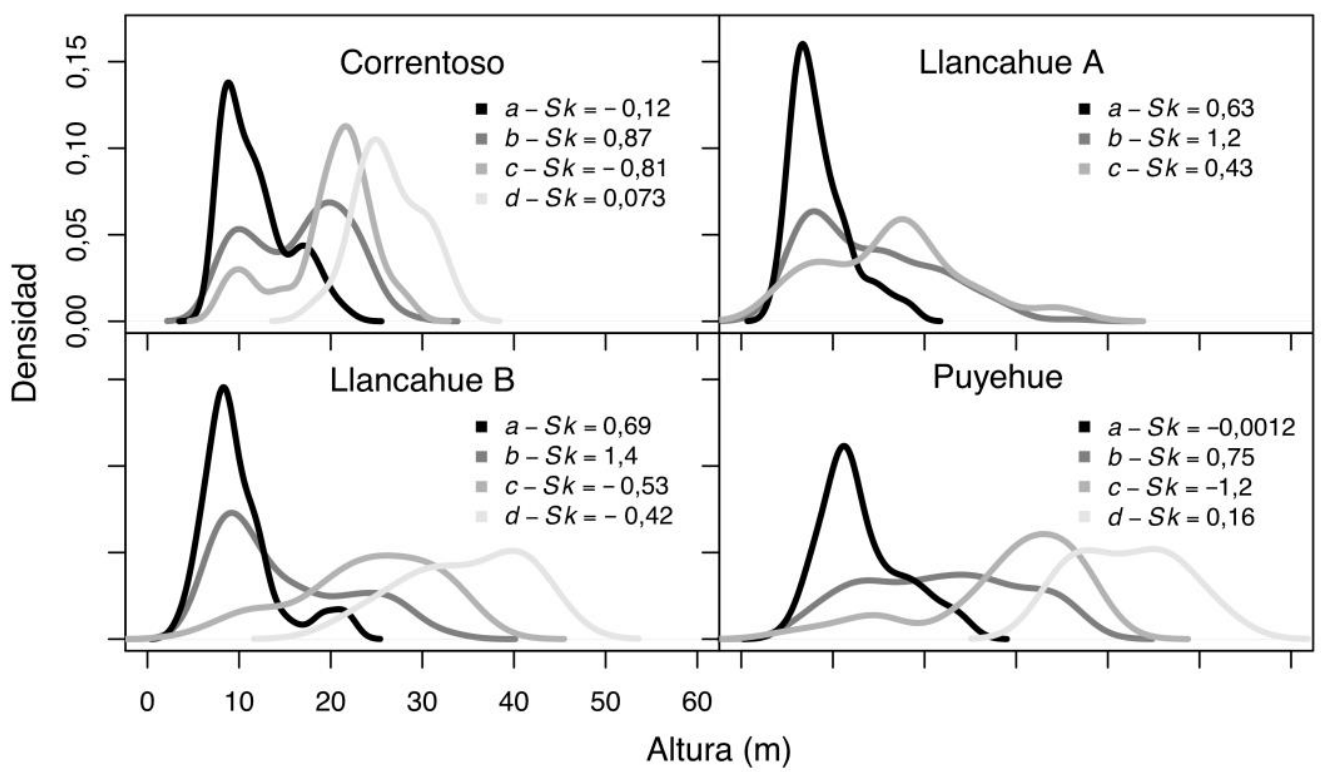

Figura 3. Distribuciones de densidad de alturas de los árboles agrupados según el análisis clúster de acuerdo a la similitud de sus distribuciones de alturas (ver también figura 2). $\mathrm{Sk}=$ valor de coeficiente de asimetría.

Density distributions of tree heights grouped using a cluster analysis according to the similarity of their distributions of heights (see also figure 2). $\mathrm{Sk}=$ value of asymmetry coefficient. 
especies de tolerancia intermedia a intolerantes (excepto Saxegothaea conspicua), y se caracterizó por presentar distribuciones de asimetría negativa (Sk cercano a -1), con excepción de Llancahue-A donde presentó un valor de Sk cercano a 0. Finalmente las especies pioneras Nothofagus dombeyi y Nothofagus nitida (grupo "d"), se posicionaron a mayor altura que las demás distribuciones, siguiendo una distribución normal (Sk cercano a 0).

\section{DISCUSIÓN}

En este estudio se determinó la existencia de dos a tres estratos en el dosel en bosques antiguos siempreverde de Chile, concordando con resultados de estratificación en bosques templados en otras regiones del mundo (Terborgh 1985, Ashton y Hall 1992, Baker y Wilson 2000). Los resultados rechazan la hipótesis $i$, ya que la dominancia de especies tolerantes en el presente estudio no propició un dosel no estratificado (i.e. heterogéneo). Multiples estratos en el dosel (i.e. tres estratos) solo se observaron de forma parchosa en el bosque (escala espacial de las parcelas) en sectores donde los árboles emergentes generan un tercer estrato. Estos árboles emergentes tienen densidad reducida en los rodales, y por lo tanto no conforman un estrato a nivel de rodal (figura 1). No obstante, los árboles emergentes aportan a la complejidad espacial del dosel (Franklin y Van Pelt 2004).

Los resultados de este trabajo no evidencian, a escala de rodal, que el mayor porcentaje de especies tolerantes a la sombra en los bosques estudiados propicien una distribución unimodal como la documentada por Gutiérrez et al. (2004). Sin embargo, es importante notar que si se observó este patrón a escalas espaciales de las parcelas. Se propone que la estructura de dosel descrita por Gutiérrez et al. (2004) difiere por las escalas espaciales de análisis, características de composición (i.e. presencia de un denso sotobosque de Tepualia stipularis), suelo (i.e. ñadis) y disturbios diferentes a la de los bosques analizados en este estudio. En este estudio el patrón de estratificación a escala de rodal ocurriría porque las distribuciones de alturas de los grupos de especies (figura 3) se diferenciaron claramente entre aquellas que ocupaban los estratos inferiores y superiores. En el caso específico de Puyehue, las especies que se agrupaban en la parte media del dosel, tenían marcadas diferencias funcionales de longevidad, tamaño máximo, y tolerancia a la sombra según los grupos de especies obtenidos del análisis clúster (cuadro 2, figura 3). A base de la hipótesis $i$, era esperable que en estos rodales se hubiera producido un recambio progresivo de las cohortes de especies pioneras, por cohortes de especies de mayor tolerancia a la sombra (Franklin et al. 2002, Franklin y Van Pelt 2004). Como ha sido observado en otros bosques templados, el aumento de individuos tolerantes provoca una reducción de estratos arbóreos (Baker y Wilson 2000). Por lo tanto, era razonable esperar que una mayor proporción de árboles tolerantes a la sombra propiciasen la for- mación de distribuciones de alturas no estratificadas, ya que pueden posicionarse en casi cualquier altura del perfil vertical del bosque (Gutiérrez et al. 2004). No obstante, a escala de rodal, los resultados documentan la presencia de un grupo de especies dominantes y emergentes (grupo c y d en figura 2) que conforman un estrato bien definido en el dosel superior, y que a raíz de su longevidad ( $>500$ años), probablemente persistan en este estrato por un largo tiempo, hasta que se produzcan disturbios a escala de rodal (e.g. incendios, deslizamientos de tierra, volteo por viento), que permitan el restablecimiento de nuevas cohortes de especies pioneras (Pollmann y Veblen 2004). Adicionalmente, los disturbios de pequeña escala espacial dentro del bosque, producidos por la caída de uno o varios árboles, crearían condiciones de heterogeneidad de luz y otros recursos (White y Pickett 1985, Franklin y Van Pelt 2004), que influirían en la formación de los estratos inferiores, compuestos por especies de menor tamaño, mayor tolerancia a la sombra y ciclos de vidas más cortos (grupos a y b en figura 2), que dan cuenta de características de adaptación de las especies a los distintos tipos de disturbios.

En concordancia con lo anterior, se observa una consistente diferenciación entre los grupos de especies a base de su ubicación en el dosel y sus respectivos rasgos funcionales (figura 3 ), sugiriendo que los grupos de especies tienen diferentes estrategias de vida, desarrolladas a partir de su adaptación a los procesos dinámicos que afectan al bosque, y que permiten la formación de estratos y la coexistencia de especies (sensu Brokaw y Busing 2000). Consecuentemente, las cuatro agrupaciones de especies en el perfil vertical presentan tipos de distribuciones de frecuencia en directa relación con sus características funcionales. Por ejemplo, el grupo "c" (figura 3) en la mayoría de los casos, presentó una distribución con asimetría negativa, a raíz de las limitaciones de las especies de este grupo para regenerar bajo doseles con condiciones de luz restringida (Donoso 1993). Las especies localizadas en el estrato emergente (grupo d), conformado por las especies pioneras $N$. dombeyi y/o N. nitida, presentaron distribuciones de altura normales por sobre las demás especies, típicas de remanentes de cohortes coetáneas y que generan un importante aporte en área basal (Donoso y Lusk 2007). El grupo correspondiente a las especies tolerantes a la sombra, tales como Aextoxicon punctatum y Laureliopsis philippiana (grupo b en figura 2), no refleja un tipo definido de distribución, pero presenta una cantidad importante de individuos tanto en la parte alta como baja del dosel, lo que posibilitaría la existencia de una estructura vertical más heterogénea en función del reemplazo de especies intolerantes en ausencia de disturbios de gran escala espacial (Franklin et al. 2002, Franklin y Van Pelt 2004, Gutiérrez y Huth 2012). Esto se observó especialmente en Puyehue donde estas especies presentaron una tendencia a ubicarse en la parte media del dosel. Consecuentemente, estos resultados apoyan la hipótesis $i i$ que la estratificación del dosel está asociada al proceso de dinámica de 
claros ya que genera un recambio de cohortes de árboles con distintas alturas máximas y tolerancia a la sombra a lo largo de la sucesión (Franklin et al. 2002, Gutiérrez y Huth 2012). Asimismo, basado en los resultados, se sugiere que en etapas tardías de la sucesión, estos estratos arbóreos no desaparecerían a escala de rodal en bosques del tipo forestal siempreverde.

Los resultados de estratificación vertical en bosques antiguos del tipo forestal siempreverde del presente estudio (cuadro 3) sugieren que existe estabilidad estructural a escala de rodal, es decir que ciertas características estructurales se mantienen entre ciertos parámetros producto de la capacidad de resistencia y recuperación de los ecosistemas tras la ocurrencia de disturbios (Perry 1994). En etapas tempranas de la sucesión de los bosques siempreverdes descritos en Chile, se han encontrado distribuciones de alturas con dos estratos (Lusk y Ortega 2003), pero con la particularidad que la diferenciación de estratos ocurre entre especies dominantes y codominantes y no por la integración de nuevos cohortes de especies tolerantes a sombra (observación personal Pablo Donoso). En los bosques antiguos siempreverdes que aquí se estudiaron se encontraron dos estratos en el dosel (cuadro 3), lo que en conjunto con los resultados de Lusk y Ortega (2003) sugerirían que la estabilidad de estratos se alcanzaría en fases tempranas de la sucesión, y se mantendría a raíz de la adaptación de las especies a los disturbios que afectan al bosque (e.g. caídas individuales de árboles). Se propone que la ocurrencia y la escala espacial de los disturbios determinarían la heterogeneidad vertical a lo largo del desarrollo de los bosques de este tipo forestal, y la estabilidad estructural explicaría la mantención de la estratificación del dosel.

En este estudio se describe la estructura vertical de bosques siempreverde andinos y costeros que tienen diferencias climáticas, edáficas, distintas historias de disturbios (e.g. bosques andinos son afectados por actividad volcánica). Estas diferencias se expresan en diferentes composiciones, alturas máximas y áreas basales. A pesar de estas diferencias, tanto la estatificación como la conformación vertical de los grupos de especies, compartieron un patrón de estratificación común y concordante con las estratificaciones encontradas en otros bosques del mundo (Terborgh 1985, Ashton y Hall 1992, Baker y Wilson 2000). Futuras investigaciones podrían enfocarse en estudiar los patrones de estrificación del dosel utilizando sensores remotos como LiDAR (inglés: Light Detection and Ranging o Laser Imaging Detection and Ranging) para establecer si el patrón aquí documentado se comparte en otros bosques nativos chilenos y como estos patrones varían en detalle a través de diversas escalas espaciales. Utilizando esta tecnología también es posible estudiar la posible estratificación del sotobosque generarada por arbustos y otras plantas que no fueron estudiadas en esta investigación. Este estudio aporta en avanzar en el entendimiento del patrón de estratificación y su vínculo con la productividad aditiva que aportan árboles emergentes en este tipo forestal (Lusk y Ortega 2003), y sus implicancias para las decisiones del manejo forestal (e.g., resiliencia a madereo selectivo; aptitud para silvicultura multietánea). Expandiendo esta investigación con análisis LiDAR aportaría en establecer la relación funcional (e.g. productividad) entre la distribución vertical de alturas de los árboles y la distribución del área foliar en estos bosques. Finalmente, los resultados de este estudio refuerzan la relevancia de la estructura del dosel para entender la provisión de múltiples hábitats en bosques antiguos, ya que se reconoce la directa relación que existe entre aves que ocupan diferenciadamente el dosel superior e inferior del bosque, y la importancia que implica para la fauna, la conformación de estos hábitats en el dosel (Díaz et al. 2005).

\section{CONCLUSIONES}

El presente estudio demuestra que el patrón predominante de distribución de alturas de árboles en el dosel de bosques antiguos siempreverde es de dos a tres estratos de dosel. Los resultados documentan que especies arbóreas de diferentes grupos funcionales conforman cada uno de los estratos, debido a la adaptación de las especies a la constante ocurrencia de disturbios (e.g. caídas individuales de árboles), resultando en estratos dominados por especies arbóreas con distinta longevidad, tolerancia a la sombra y tamaño. Sin embargo, la demanda lumínica de las especies por sí sola no explicó la estratificación de los bosques estudiados. Se propone que la dinámica de claros y la estabilidad estructural a lo largo del desarrollo de los bosques explican los resultados de estratificación del dosel encontrados. Esto provee un marco conceptual para abordar cómo los mecanismos y procesos dinámicos del bosque influyen en la estructuración vertical del dosel, la conformación de estratos del dosel dominados por diferentes estrategias de vida de las especies, y la consiguiente coexistencia de estas. Los resultados documentados en este estudio, sobre los patrones de agregación de copas, aportan en el entendimiento de la distribución y estratificación del dosel arbóreo de los bosques antiguos del tipo forestal siempreverde, lo que tiene una potencial relevancia para el manejo sostenible y la conservación de la biodiversidad de estos ecosistemas.

\section{AGRADECIMIENTOS}

Agradecemos a Ignacio Díaz-Hormazábal por asistencia en R, a Esteban Arias, Andrés Lara, Romina Novoa y Juan Pablo Cárdenas, por el apoyo en las campañas de recolección de datos. Además, PJD agradece al proyecto FONDECYT N ${ }^{\circ} 1150496$, y AGG al Fondo de Investigación del Bosque Nativo 27/2015. Agradecemos a dos revisores anónimos por sus constructivas revisiones para mejorar este trabajo. 


\section{REFERENCIAS}

Ashton PS, P Hall. 1992. Comparisons of structure among mixed dipterocarp forests of north-western Borneo. Journal of Ecology 80: 459-481. DOI: 10.2307/2260691

Baker PJ, JS Wilson. 2000. A quantitative technique for the identification of canopy stratification in tropical and temperate forests. Forest Ecology and Management 127:77-86. DOI: $\underline{10.1016 / \mathrm{S} 0378-1127(99) 00118-8}$

Brokaw NV, RT Busing. 2000. Niche versus chance and tree diversity in forest gaps. Trends in Ecology and Evolution 15: 183-188. DOI: $10.1016 / \mathrm{S} 0169-5347(00) 01822-\mathrm{X}$

Díaz IA, JJ Armesto, S Reid. 2005. Linking forest structure and composition: avian diversity in successional forests of Chiloe' Island, Chile. Biological Conservation 123: 91-101. DOI: 10.1016/j.biocon.2004.10.011

Donoso C. 1993. Bosques templados de Chile y Argentina. Variación, estructura y dinámica. Santiago, Chile. Editorial Universitaria. $483 \mathrm{p}$.

Donoso PJ, CH Lusk. 2007. Differential effects of emergent Nothofagus dombeyi on growth and basal area of canopy species in an old-growth temperate rainforest. Jounal of Vegetation Science 18: 675-684. DOI: 10.1111/j.16541103.2007.tb02581.x

Franklin JF, R Van Pelt. 2004. Spatial aspects of structural complexity in old-growth forests. Journal of Forestry 102: 2228. DOI: $10.1093 /$ jof $/ 102.3 .22$

Franklin JF, TA Spies, R van Pelt, A Carey, D Thornburgh, DR Berg, DB Lindenmayer, M Harmon, W Keeton, DC Shaw. 2002. Disturbances and the structural development of natural forest ecosystems with some implications for silviculture. Forest Ecology and Management 155: 399-423. DOI: $\underline{10.1016 / \mathrm{S} 0378-1127(01) 00575-8}$

Gutiérrez AG, A Huth. 2012. Successional stages of primary temperate rainforests of Chiloé Island, Chile. Perspectives in Plant Ecology, Evolution and Systimatics 14: 243-256. DOI: $10.1016 /$ j.ppees.2012.01.004

Gutiérrez AG, JJ Armesto, JC Aravena. 2004. Disturbance and regeneration dynamics of an old-growth North Patagonian rain forest in Chiloe Island, Chile. Journal of Ecology 92: 598-608. DOI: https://doi.org/10.1111/j.0022$\underline{0477.2004 .00891 . x}$

Gutiérrez AG, JJ Armesto, JC Aravena, NV Carrasco, DA Christie, MR Carmona, C Pérez, PM Peña, A Huth. 2009. Structural and environmental characterization of old-growth temperate rainforests of northern Chiloe Island, Chile: regional and global relevance. Forest Ecology and Management 258: 376-388. DOI: 10.1016/j.foreco.2009.03.011
Hardiman BS, CM Gough, A Halperin, K Hofmeister, LE Nave, G Bohrer, PS Curtis. 2013. Maintaining high rates of carbon storage in old forests: a mechanism linking canopy structure to forest function. Forest Ecology and Management 298:111-119. DOI: 10.1016/j.foreco.2013.02.031

Komárek A. 2009. A new R package for Bayesian estimation of multivariate normal mixtures allowing for selection of the number of components and interval-censored data. Computational Statistics and Data Analysis 53: 3932-3947. DOI: 10.1016/j.csda.2009.05.006

Lusk C, A Ortega. 2003. Vertical structure and basal area development in second-growth Nothofagus stands in Chile. Journal of Applied Ecology 40: 639-645. DOI: 10.1046/j.13652664.2003.00827.x

McLachlan G, D Peel. 2000. Finite Mixture Models. New York, USA. Wiley. 419 p.

Parker GG. 1997. Canopy structure and light environment of an old-growth Douglas-fir/western hemlock forest. Northwest Science 71: 261-270.

Perry DA. 1994. Forest ecosystems. Baltimore, USA. Johns Hopkins University Press. 649 p.

Pollmann W, TT Veblen. 2004. Nothofagus regeneration dynamics in Southcentral Chile: A test of a general model. Ecological Monographs 74 (4): 615-634. DOI: 10.1890/04-0004

Oliver CD, BC Larson. 1996. Forest Stand Dynamics. New York, USA. Wiley. 544 p.

R Development Core Team. 2017. R: A language and environment for statistical computing. Version 3.4.1. R Foundation for Statistical Computing, Vienna, Austria.

Terborgh J. 1985. The vertical component of plant species diversity in temperate and tropical forests. American Naturalist 126: 760-777. DOI: $10.1086 / 284452$

Watson, JM, T Evans, O Venter, B Williams, A Tulloch, C Stewart, I Thompson, JC Ray, K Murray, A Salazar, C McAlpine, P Potapov, J Walston, JG Robinson, M Painter, D Wilkie, C Filardi, WF Laurance, RA Houghton, S Maxwell, H Grantham, C Samper, S Wang, L Laestadius, RK Runting, GA Silva-Chávez, J Ervin, D Lindenmayer. 2018. The exceptional value of intact forest ecosystems. Nature Ecology \& Evolution 2(4): 599-610. DOI: 10.1038/ s41559-018-0490-x

White PS, STA Pickett. 1985. Natural disturbance and Patch dynamics: An introduction. In Pickett STA, PS White eds. The ecology of natural disturbance and patch dynamics. San Diego, USA. Academic Press. p. 3-13.

Xu L, EJ Bedrick, T Hanson, C Restrepo. 2014. A comparison of statistical tools for identifying modality in body mass distributions. Journal of Data Science 12: 175-196. 